TRANSACTIONS OF THE

AMERICAN MATHEMATICAL SOCIETY

Volume 355, Number 8, Pages 3065-3077

S 0002-9947(03)03221-5

Article electronically published on March 19, 2003

\title{
WEIERSTRASS FUNCTIONS WITH RANDOM PHASES
}

\author{
YANICK HEURTEAUX
}

Abstract. Consider the function

$$
f_{\theta}(x)=\sum_{n=0}^{+\infty} b^{-n \alpha} g\left(b^{n} x+\theta_{n}\right)
$$

where $b>1,0<\alpha<1$, and $g$ is a non-constant 1-periodic Lipschitz function. The phases $\theta_{n}$ are chosen independently with respect to the uniform probability measure on $[0,1]$. We prove that with probability one, we can choose a sequence of scales $\delta_{k} \searrow 0$ such that for every interval $I$ of length $|I|=\delta_{k}$, the oscillation of $f_{\theta}$ satisfies $\operatorname{osc}\left(f_{\theta}, I\right) \geq C|I|^{\alpha}$. Moreover, the inequality $\operatorname{osc}\left(f_{\theta}, I\right) \geq C|I|^{\alpha+\varepsilon}$ is almost surely true at every scale. When $b$ is a transcendental number, these results can be improved: the minoration $\operatorname{osc}\left(f_{\theta}, I\right) \geq C|I|^{\alpha}$ is true for every choice of the phases $\theta_{n}$ and at every scale.

\section{INTRODUCTION}

The function

$$
w(x)=\sum_{n=0}^{+\infty} b^{-n \alpha} \cos \left(2 \pi b^{n} x\right),
$$

where $b>1$ and $\alpha \leq 1$, is probably one of the most famous continuous nowhere differentiable functions. This function was introduced by Weierstrass. He proved that $w$ is nowhere differentiable for some of these values $b$ and $\alpha$. A few years later, Hardy gave the proof for every $b>1$ and every $\alpha \leq 1$ (see [5]).

More generally, one can consider the function

$$
f(x)=\sum_{n=0}^{+\infty} b^{-n \alpha} g\left(b^{n} x\right),
$$

where $g$ is a 1 -periodic Lipschitz function, $1<b<+\infty$ and $0<\alpha<1$. Such a function will be called a Weierstrass function, and it is easy to prove that $f$ is of class $C^{\alpha}$ (see, for example, 3]). Note that the regularity property of $f$ is more subtle when $\alpha=1$. In that case, $f$ lies in the Zygmund class but is often not Lipschitz.

A famous conjecture states that the Hausdorff dimension of the graph of $f$ is equal to $2-\alpha$ (this is the biggest value that one can hope for). There are many papers that give general support to this conjecture (see, for example, [11, [10], 6], [7], 9], 14], 15]). Of course, one cannot expect this conjecture to be true in

Received by the editors July 8, 2002.

2000 Mathematics Subject Classification. Primary 26A27, 28A80, 37A05; Secondary 60F20.

Key words and phrases. Weierstrass functions, almost periodic functions, oscillations, fractal dimension. 
general. Suppose for instance that $g$ is of the form $g(x)=\varphi(x)-b^{-\alpha} \varphi(b x)$ with $\varphi$ a smooth function. Then $f=\varphi$, and the graph has dimension 1 . Thus, the genuine question is whether the conjecture is true when $g$ is not of the above form. In recent work with Thierry Bousch, we proved the following result about the oscillations of the function $f$, which supports the conjecture.

Theorem 1.1 ([2], [1]). Let $g$ be a 1-periodic Lipschitz function, $1<b<+\infty$ and $0<\alpha<1$. Define $f$ using formula (1.1). There are only two possible mutually exclusive cases:

(i) $f$ is Lipschitz and $\left\|f^{\prime}\right\|_{\infty} \leq \frac{\left\|g^{\prime}\right\|_{\infty}}{b^{1-\alpha}-1}$,

or

(ii) there exists a constant $C>0$ such that for every interval I of length $|I| \leq 1$,

$$
\operatorname{osc}(f, I)=\sup _{I}(f)-\inf _{I}(f) \geq C|I|^{\alpha} .
$$

Moreover, the set of functions $g$ such that (ii) is satisfied is a dense open subset of the space of 1-periodic Lipschitz functions (equipped with its standard norm).

The proof of this result is based on the following functional equation:

$$
f(x)=g(x)+b^{-\alpha} f(b x)
$$

which is satisfied by the Weierstrass function $f$. Let us also recall that conclusion (1.2) is often present in the literature about Weierstrass-type functions. In [14, sufficient conditions on $g$ are given which ensure that (1.2) is satisfied. Assuming some stronger properties on $g$, Kaplan et al. (9]) prove a result similar to Theorem 1.1 about the behavior of $f$. Finally, in [7], Hu and Lau prove a conclusion slightly different from (1.2) in the case where the Weierstrass-Mandelbrot function associated with $f$ is not identically equal to zero.

It is well known that conclusion (1.2) ensures that the box-counting dimension of the graph of $f$ is greater than $2-\alpha$ (see, for example, [3]). On the other hand, McMullen shows in [12] that there are self-affine functions of class $C^{\alpha}$ satisfying (1.2) but whose graphs have Hausdorff dimension strictly less than $2-\alpha$ (see also 14]). These functions are not Weierstrass-type functions and do not refute the conjecture.

The purpose of this paper is to study the local oscillation behavior of Weierstrass functions with phases. Such a function will be defined by the formula

$$
f_{\theta}(x)=\sum_{n=0}^{+\infty} b^{-n \alpha} g\left(b^{n} x+\theta_{n}\right)
$$

where $g$ is a 1-periodic Lipschitz function, $1<b<+\infty, 0<\alpha<1$ and $\theta=\left(\theta_{n}\right)_{n \geq 0}$ is a sequence of phases satisfying $\theta_{n} \in[0,1]$. It is easy to check that the function $f_{\theta}$ is still Hölder continuous on $\mathbb{R}$ with exponent $\alpha$ (see, for example, [3] or [8]). More precisely, one can prove that the Hölder constant $C$ of $f_{\theta}$ satisfies

$$
C \leq\left[\frac{\left\|g^{\prime}\right\|_{\infty}}{1-b^{\alpha-1}}+\frac{\operatorname{osc}(g)}{1-b^{-\alpha}}\right],
$$

where $\operatorname{osc}(g)=\sup _{\mathbb{R}}(g)-\inf _{\mathbb{R}}(g)$ is the global oscillation of the function $g$. In particular, this constant does not depend on $\theta$.

When $b$ is an integer, the function $f_{\theta}$ is 1-periodic. In the other cases we can only ensure that $f_{\theta}$ is almost periodic (as a uniform limit of a sequence of almost 


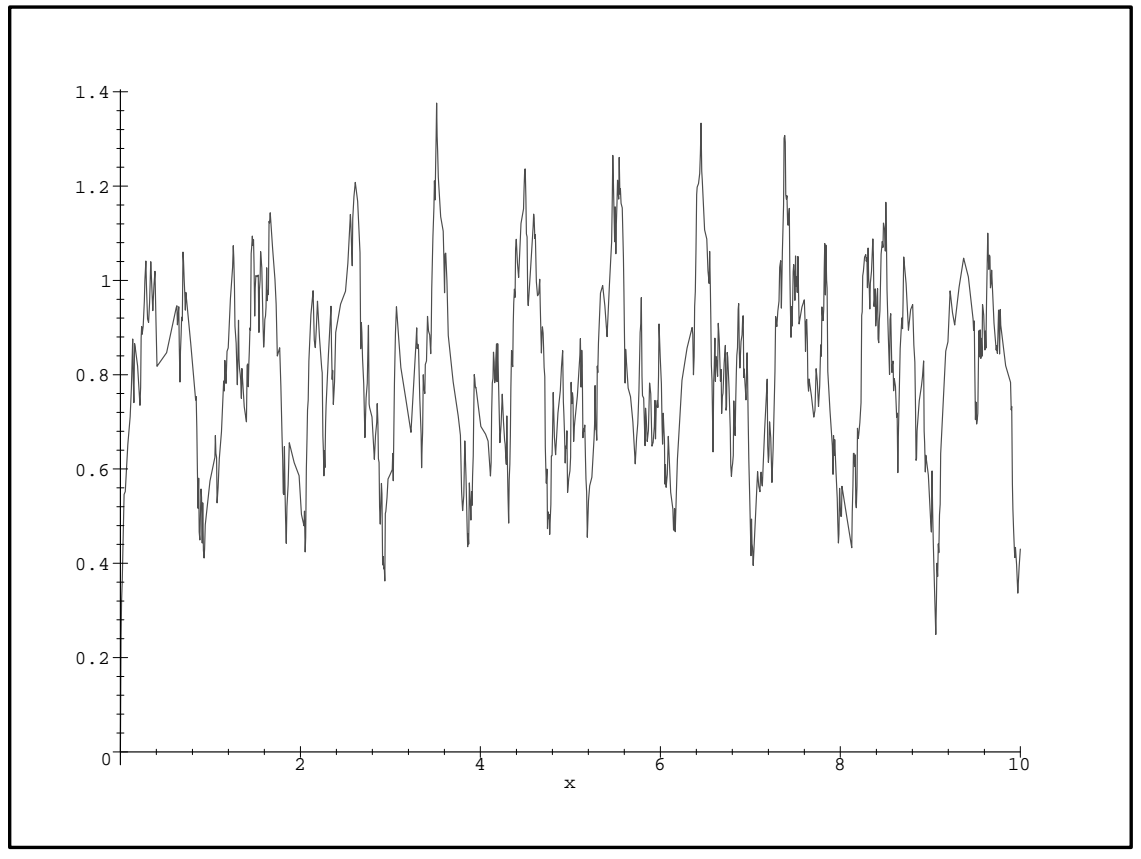

Figure 1. Graph of $f_{\theta}(x)$ with $g(x)=\operatorname{dist}(x, \mathbb{Z}), b=2.1$ and $\alpha=0.5$

periodic functions). More precisely, we can establish the following property, which states that the almost periodicity property does not depend on the sequence of phases $\theta$. Such a property will be useful in the proof of the main theorem (see Lemma 4.2.

Proposition 1.2 (Equi almost periodicity). The family of functions $\left(f_{\theta}\right)_{\theta \in[0,1]^{\mathbb{N}}}$ satisfies the following property:

$$
\forall \varepsilon>0, \exists \ell>0 ; \forall \theta \in[0,1]^{\mathbb{N}}, \forall \delta \in \mathbb{R}, \exists a \in[\delta, \delta+\ell) ;\left\|\tau_{a} f_{\theta}-f_{\theta}\right\|_{\infty} \leq \varepsilon
$$

$\left(\tau_{a} f_{\theta}\right.$ is defined by $\left.\tau_{a} f_{\theta}(x)=f_{\theta}(x+a)\right)$.

Proof. This proposition is a consequence of the compactness of the set $[0,1]^{\mathbb{N}}$. Let $\varepsilon>0$ and $\theta \in[0,1]^{\mathbb{N}}$. We know that the function $f_{\theta}$ is almost periodic. So, we can find $\ell_{\theta}>0$ such that for all $\delta \in \mathbb{R}$, there exists $a_{\theta} \in\left[\delta, \delta+\ell_{\theta}\right)$ with $\left\|\tau_{a_{\theta}} f_{\theta}-f_{\theta}\right\|_{\infty} \leq \varepsilon / 3$. The application $\theta \in[0,1]^{\mathbb{N}} \mapsto f_{\theta} \in \mathcal{C}_{b}(\mathbb{R})$ being continuous (when the set $\mathcal{C}_{b}(\mathbb{R})$ of bounded continuous functions is endowed with the norm \|\|$\left._{\infty}\right)$, we can find $\theta^{1}, \ldots, \theta^{n} \in[0,1]^{\mathbb{N}}$ such that the set $\left\{f_{\theta}\right\}$ is covered by the balls $B\left(f_{\theta^{k}}, \varepsilon / 3\right)$. Let $\ell=\max \left(\ell_{\theta^{1}}, \ldots, \ell_{\theta^{n}}\right)$. If $\delta \in \mathbb{R}$ and if $f_{\theta} \in B\left(f_{\theta^{k}}, \varepsilon / 3\right)$, we obtain $\left\|\tau_{a_{\theta k}} f_{\theta}-f_{\theta}\right\|_{\infty} \leq \varepsilon$. The conclusion follows if we note that $a_{\theta^{k}} \in[\delta, \delta+\ell)$.

As we can see in Figure 1, the function $f_{\theta}$ seems to be irregular, and we would like to know if a minoration similar to (1.2) is true for Weierstrass functions with phases. In the general case, such a minoration seems to be difficult to obtain for each value of the sequence $\theta=\left(\theta_{n}\right)_{n \geq 0}$. That is the reason why we propose to 
consider the phases $\theta_{n}$ as independent random variables uniformly distributed in $[0,1]$. In other words, the set of phases $\Omega=[0,1]^{\mathbb{N}}$ is endowed with the probability measure $\mathbb{P}=d x^{\otimes \mathbb{N}}$, where $d x$ denotes the Lebesgue measure on $[0,1]$. Recall that in such a context, B. Hunt proved that when $g(x)=\cos x$, the Hausdorff dimension of the graph of the function $f_{\theta}$ is equal to $2-\alpha$ with probability one (see [8]). Using a different method, Szulga recently proved a similar result for the WeierstrassMandelbrot process (16]).

If we want to write a functional equation for the function $f_{\theta}$, we also have to introduce the shift operator on $\Omega$ :

$$
T:\left(\theta_{n}\right)_{n \geq 0} \in \Omega \mapsto\left(\theta_{n+1}\right)_{n \geq 0} \in \Omega .
$$

Then, the functional equation can be written as follows:

$$
f_{\theta}(x)=g\left(x+\theta_{0}\right)+b^{-\alpha} f_{T \theta}(b x) .
$$

It is clear that the probability $\mathbb{P}$ is $T$-invariant. Moreover, the $0-1$ law, which is satisfied by the independent phases, states that the $\sigma$-field $B_{\infty}=\bigcap_{n=0}^{\infty} T^{-n}\left(B_{0}\right)$ is constituted of events with trivial probability ( $B_{0}$ is the $\sigma$-field of Borel sets on $\Omega$ ). Then, it is classical to conclude that the strong mixing property

$$
\forall A, B \in B_{0}, \quad \lim _{n \rightarrow \infty} \mathbb{P}\left[A \cap T^{-n} B\right]=\mathbb{P}[A] \mathbb{P}[B]
$$

is satisfied by the $T$-invariant probability $\mathbb{P}$ (for elementary results on ergodic theory, see for example [13] or [19]). In particular, the probability $\mathbb{P}$ is ergodic: invariant Borel sets have probability 0 or 1 . This ergodicity will be the key point of the proof of our main theorems (Theorems 3.1 and 3.2 ).

Let us now briefly describe the main results of this paper. We first observe in Section 2 that a conclusion like (i) in Theorem 1.1 is not possible in the random context. More precisely, when $g$ is not constant, $f_{\theta}$ is almost surely not Lipschitz. Then, we can state in Section 3 and prove in Section 4 minorations for the oscillations. More precisely, we prove that with probability one, the minoration

$$
\operatorname{osc}\left(f_{\theta}, I\right) \geq C|I|^{\alpha+\varepsilon}
$$

is true for every interval $I$, as soon as $g$ is not constant. Moreover, the stronger inequality

$$
\operatorname{osc}\left(f_{\theta}, I\right) \geq C|I|^{\alpha}
$$

is valid when $|I|=\delta_{n}$, where $\left(\delta_{n}\right)_{n}$ is a sequence of scales decreasing to zero. In particular, the local Hölder index of $f_{\theta}$ is almost surely everywhere equal to $\alpha$. In Section 5, we deal with the case where $b$ is a transcendental number. In that case, we are able to prove that the minoration (1.8) is true for every interval $I$ and for every choice of phases $\left(\theta_{n}\right)_{n \geq 0}$. In some sense, this means that randomness is already present in the number $b$.

\section{When $g$ is nOt CONSTANT, $f_{\theta}$ IS NOt Lipschitz}

The first step of our investigation is to establish that $f_{\theta}$ cannot be regular (except when $g$ is constant). This is the aim of the following result.

Theorem 2.1. Let $g: \mathbb{R} \rightarrow \mathbb{R}$ be a 1-periodic Lipschitz function, $1<b<+\infty$ and $0<\alpha<1$. Define $f_{\theta}$ by formula (1.4), $c_{0}=\frac{\left\|g^{\prime}\right\|_{\infty}}{b^{1-\alpha}-1}$, and let

$$
A=\left\{\theta \in \Omega ; f_{\theta} \text { is Lipschitz }\right\} \quad \text { and } A_{c_{0}}=\left\{\theta \in \Omega ; f_{\theta} \text { is } c_{0} \text {-Lipschitz }\right\} \text {. }
$$


Then,

$$
\mathbb{P}[A]=0 \text { or } 1 \quad \text { and } \quad \mathbb{P}\left[A_{c_{0}}\right]=0 \text { or } 1 .
$$

Moreover, the following are equivalent:

(i) $\mathbb{P}[A]=1$;

(ii) $\mathbb{P}\left[A_{c_{0}}\right]=1$;

(iii) $g$ is a constant function.

Remark 2.2. If $g$ is not constant, Theorem 2.1 ensures that with probability one, there exist $x \neq y$ and $v>0$ such that

$$
\left|f_{\theta}(x)-f_{\theta}(y)\right| \geq \frac{(1+v)\left\|g^{\prime}\right\|_{\infty}}{b^{1-\alpha}-1}|x-y| .
$$

In fact, the theorem also says that there is no more information once one shows that $f_{\theta}$ is almost surely not Lipschitz. This remark will be useful when proving Theorem 3.1.

Proof of Theorem 2.1. Observing the functional equation (1.6), it is clear that $A$ is $T$-invariant. The ergodicity of the shift $T$ then ensures that $\mathbb{P}[A]=0$ or $\mathbb{P}[A]=1$. More precisely, if $A_{c}=\left\{\theta \in \Omega ; f_{\theta}\right.$ is $c$-Lipschitz $\}$ and $\varphi(c)=b^{\alpha-1}\left(c+\left\|g^{\prime}\right\|_{\infty}\right)$, it is easy to check that

$$
\theta \in A_{c} \Longrightarrow T \theta \in A_{\varphi(c)}
$$

In other words,

$$
A_{c} \subset T^{-1}\left(A_{\varphi(c)}\right)
$$

In particular, if $c \geq c_{0}$, then $\varphi(c) \leq c$ and $A_{c} \subset T^{-1}\left(A_{c}\right)$. We cannot state that $A_{c}=T^{-1}\left(A_{c}\right)$, but we can however conclude that $\mathbb{P}\left[A_{c}\right]=0$ or $\mathbb{P}\left[A_{c}\right]=1$. This well-known result is a consequence of the fact that an ergodic invariant probability measure has no wandering set of positive measure. Let us propose an elementary direct proof, in order to be self-contained.

Lemma 2.3. Let $B \subset \Omega$ be a Borel set such that $B \subset T^{-1} B$. Then

$$
\mathbb{P}[B]=0 \quad \text { or } \quad \mathbb{P}[B]=1 .
$$

Proof. Let $C=\Omega \backslash B$ and $D=\lim \sup _{n \rightarrow \infty} T^{-n} C$. Observe that $D$ is $T$-invariant, and suppose that $\mathbb{P}[B]<1$. Then $\mathbb{P}[D]>0$ and, by ergodicity, $\mathbb{P}[D]=1$ (here, we use the fact that $\mathbb{P}$ is a finite measure). It follows that $\mathbb{P}[B]=\mathbb{P}[B \cap D]$. But, if $B \subset T^{-1} B$, then $B \cap D=\emptyset$. So $\mathbb{P}[B]=0$.

Proof of (i) $\Rightarrow$ (ii). Suppose that $\mathbb{P}[A]=1$ and note that $A=\bigcup_{c>c_{0}} A_{c}$. So, we can find some $c \geq c_{0}$ such that $\mathbb{P}\left[A_{c}\right]>0$. It follows that $\mathbb{P}\left[A_{c}\right]=1$. Using (2.1) and the $T$-invariance of the measure $\mathbb{P}$, we obtain $\mathbb{P}\left[A_{\varphi(c)}\right]=1$. Iterating this, we get $\mathbb{P}\left[A_{\varphi^{n}(c)}\right]=1$. Taking the limit when $n \rightarrow \infty$, we conclude that $\mathbb{P}\left[A_{c_{0}}\right]=1\left(c_{0}\right.$ is the unique fixed point of the contraction $\varphi$ ).

Proof of (ii) $\Rightarrow$ (iii). Let $x$ and $y$ be two real numbers, and write

$$
f_{\theta}(x)-f_{\theta}(y)=\sum_{n=0}^{+\infty} b^{-n \alpha}\left(g\left(b^{n} x+\theta_{n}\right)-g\left(b^{n} y+\theta_{n}\right)\right) .
$$


The random variables $b^{-n \alpha}\left(g\left(b^{n} x+\theta_{n}\right)-g\left(b^{n} y+\theta_{n}\right)\right)$ are centered and independent (here, we use the fact that $g$ is 1-periodic). So, they are orthogonal in $L^{2}(\mathbb{P})$. If $p \geq 0$, the Pythagorean theorem ensures that

$$
\begin{aligned}
\mathbb{E}\left[\left(f_{\theta}(x)-f_{\theta}(y)\right)^{2}\right] & =\sum_{n=0}^{+\infty} b^{-2 n \alpha} \mathbb{E}\left[\left(g\left(b^{n} x+\theta_{n}\right)-g\left(b^{n} y+\theta_{n}\right)\right)^{2}\right] \\
& \geq b^{-2 p \alpha} \mathbb{E}\left[\left(g\left(b^{p} x+\theta_{p}\right)-g\left(b^{p} y+\theta_{p}\right)\right)^{2}\right] .
\end{aligned}
$$

Replacing $x$ by $b^{-p} x$ and $y$ by $b^{-p} y$ and using (ii), we get

$$
\int_{0}^{1}(g(x+t)-g(y+t))^{2} d t \leq b^{2 p \alpha} c_{0}^{2}\left|b^{-p} x-b^{-p} y\right|^{2}
$$

Taking the limit when $p \rightarrow \infty$, we obtain

$$
\int_{0}^{1}(g(x+t)-g(y+t))^{2} d t=0 .
$$

This implies that $g(x)=g(y)$.

\section{On oscillations of $f_{\theta}$}

In this section, we state the main results concerning the local behavior of Weierstrass functions with random phases.

Theorem 3.1. Let $g: \mathbb{R} \rightarrow \mathbb{R}$ be a 1-periodic non-constant Lipschitz function, and let $1<b<+\infty$ and $0<\alpha<1$. Define $f_{\theta}$ by formula (1.4). There exists a constant $C>0$ such that for almost every $\theta \in \Omega$, we can choose a sequence of scales $\delta_{k} \searrow 0$ such that, for every interval I of length $|I|=\delta_{k}$,

$$
\operatorname{osc}\left(f_{\theta}, I\right) \geq C|I|^{\alpha} \text {. }
$$

It seems curious that we have to restrict the conclusion to some sequence of scales. In fact, it is easy to see that the more precise conclusion

$$
\exists C>0 \text {; for almost every } \theta \in \Omega, \forall I \text { with }|I| \leq 1, \operatorname{osc}\left(f_{\theta}, I\right) \geq C|I|^{\alpha}
$$

is false in general. Indeed, the set of $\theta \in \Omega$ such that $\operatorname{osc}\left(f_{\theta}, I\right) \geq C|I|^{\alpha}$ for all intervals $I$ of length $|I| \leq 1$ is clearly a closed subset of $\Omega$. So, conclusion (3.1) would imply the same property for every $\theta \in \Omega$. On the other hand, if $b$ is an integer and if $g(x)=\varphi(x)-b^{-\alpha} \varphi(b x)$, with $\varphi$ a 1-periodic non-constant Lipschitz function, then $g$ is a 1-periodic non-constant Lipschitz function. Moreover, $f_{\theta}=\varphi$ with $\theta=(0,0, \cdots)$. So we can find some $\theta \in \Omega$ such that $f_{\theta}$ is regular. As we will see in section 5 this phenomenon cannot occur when $b$ is a transcendental number.

In view of this remark, it is reasonable to ask whether the weaker assertion

$$
\text { for almost every } \theta \in \Omega, \exists C>0 ; \forall I \text { with }|I| \leq 1, \operatorname{osc}\left(f_{\theta}, I\right) \geq C|I|^{\alpha}
$$

is true when $g$ is non-constant. In fact, we do not know if (3.2) is true for every $b>1$, but we can prove an analogue of (3.2), replacing $\alpha$ by $\alpha+\varepsilon$. This is the purpose of the following theorem.

Theorem 3.2. Let $g: \mathbb{R} \rightarrow \mathbb{R}$ be a 1-periodic non-constant Lipschitz function, and let $1<b<+\infty$ and $0<\alpha<1$. Define $f_{\theta}$ by formula (1.4). For almost every $\theta \in \Omega$, the following conclusion is true:

$$
\forall \varepsilon>0, \exists C>0 ; \forall I \text { with }|I| \leq 1, \operatorname{osc}\left(f_{\theta}, I\right) \geq C|I|^{\alpha+\varepsilon} .
$$


Using Theorem 3.2 and the fact that $f_{\theta}$ is $\alpha$-Hölder, we obtain the following direct consequence about the local behavior of Weierstrass functions with phases.

Corollary 3.3. Suppose that $g$ is not constant. The function $f_{\theta}$ is almost surely nowhere differentiable. More precisely, for almost every $\theta \in \Omega$, we have

$$
\lim _{x \in I,|I| \searrow 0} \frac{\ln \left(\operatorname{osc}\left(f_{\theta}, I\right)\right)}{\ln (|I|)}=\alpha \quad \text { for all } x \in \mathbb{R} .
$$

In particular, for almost every $\theta \in \Omega$, the Hölder index of $f_{\theta}$ is everywhere equal to $\alpha$.

\section{Proof of Theorems 3.1 AND 3.2}

We begin with the following elementary lemma.

Lemma 4.1 (Transfer lemma). Let $x, y \in \mathbb{R}$ satisfy

$$
\frac{\left|f_{T \theta}(y)-f_{T \theta}(x)\right|}{|y-x|} \geq \frac{(1+v)\left\|g^{\prime}\right\|_{\infty}}{b^{1-\alpha}-1} \text { for some } v>0 \text {. }
$$

Then

$$
\frac{\left|f_{\theta}\left(b^{-1} y\right)-f_{\theta}\left(b^{-1} x\right)\right|}{\left|b^{-1} y-b^{-1} x\right|} \geq \frac{\left(1+v b^{1-\alpha}\right)\left\|g^{\prime}\right\|_{\infty}}{b^{1-\alpha}-1} .
$$

Proof. The above lemma is an easy consequence of the functional equation (1.6). We have

$$
f_{\theta}\left(b^{-1} y\right)-f_{\theta}\left(b^{-1} x\right)=g\left(b^{-1} y+\theta_{0}\right)-g\left(b^{-1} x+\theta_{0}\right)+b^{-\alpha}\left(f_{T \theta}(y)-f_{T \theta}(x)\right) .
$$

Suppose that $x, y$ and $v$ satisfy the hypothesis of the lemma. Then

$$
\begin{aligned}
\frac{\left|f_{\theta}\left(b^{-1} y\right)-f_{\theta}\left(b^{-1} x\right)\right|}{\left|b^{-1} y-b^{-1} x\right|} & \geq b^{1-\alpha} \frac{\left|f_{T \theta}(y)-f_{T \theta}(x)\right|}{|y-x|}-\frac{\left|g\left(b^{-1} y+\theta_{0}\right)-g\left(b^{-1} x+\theta_{0}\right)\right|}{\left|b^{-1} y-b^{-1} x\right|} \\
& \geq b^{1-\alpha} \frac{(1+v)\left\|g^{\prime}\right\|_{\infty}}{b^{1-\alpha}-1}-\left\|g^{\prime}\right\|_{\infty} \\
& =\frac{\left(1+v b^{1-\alpha}\right)\left\|g^{\prime}\right\|_{\infty}}{b^{1-\alpha}-1} .
\end{aligned}
$$

When we apply Lemma 4.1 we shrink the scale by a factor $1 / b$ and obtain a stronger estimate (the constant $v$ is replaced by the bigger one $v b^{1-\alpha}$ ). Using iterations of Lemma 4.1 it is possible, from an estimate of the oscillation of an iterate $f_{T^{n} \theta}$ at a big scale, to get an estimate of the oscillation of $f_{\theta}$ at a small scale. Of course, this final minoration will make sense if we are able to control the distance $|y-x|$ when we begin to use Lemma 4.1. The following lemma is a step in this direction.

Lemma 4.2. Let $v>0$. If $h, \ell>0$, denote by $M_{h, \ell}$ the set of $\theta \in \Omega$ such that for every closed interval $I$ of length $|I|=\ell$, there exists $x \in I$ with $x+h \in I$ and

$$
\left|f_{\theta}(x+h)-f_{\theta}(x)\right| \geq \frac{(1+v)\left\|g^{\prime}\right\|_{\infty}}{b^{1-\alpha}-1}|h| .
$$

Then there exist $h_{0}>0$ and $\ell_{0} \geq 1$ such that

$$
\mathbb{P}\left[M_{h_{0}, \ell_{0}}\right]>0 \text {. }
$$


Proof. Let $v>0$ and suppose that $g$ is not constant (this is the only interesting case). According to Theorem 2.1 the set

$$
M=\left\{\theta \in \Omega ; \exists x \in \mathbb{R}, \exists h>0 ;\left|f_{\theta}(x+h)-f_{\theta}(x)\right| \geq \frac{(1+3 v)\left\|g^{\prime}\right\|_{\infty}}{b^{1-\alpha}-1}|h|\right\}
$$

is such that $\mathbb{P}[M]=1$. We can then find $h_{0}>0$ such that the set $M_{h_{0}}$ of $\theta$ satisfying

$$
\left|f_{\theta}\left(x_{\theta}+h_{0}\right)-f_{\theta}\left(x_{\theta}\right)\right| \geq \frac{(1+3 v)\left\|g^{\prime}\right\|_{\infty}}{b^{1-\alpha}-1}\left|h_{0}\right| \quad \text { for some } x_{\theta} \in \mathbb{R}
$$

has positive probability. Let

$$
\varepsilon=\frac{v\left\|g^{\prime}\right\|_{\infty}}{b^{1-\alpha}-1}\left|h_{0}\right| .
$$

According to Proposition 1.2, we can find a real number $\ell_{0}>0$ (which can be supposed greater than 1 and greater than $2 h_{0}$ ) such that

$$
\forall \theta \in \Omega, \forall \delta \in \mathbb{R}, \exists a \in\left[\delta, \delta+\ell_{0} / 2\right) ;\left\|\tau_{a} f_{\theta}-f_{\theta}\right\|_{\infty} \leq \varepsilon .
$$

Let $I=\left[z, z+\ell_{0}\right]$ be a closed interval of length $\ell_{0}$. Suppose that $\theta$ satisfies (4.1) and take $\delta=z-x_{\theta}$. We can find $a \in\left[\delta, \delta+\ell_{0} / 2\right)$ such that

$$
\left\|\tau_{a} f_{\theta}-f_{\theta}\right\|_{\infty} \leq \varepsilon \text {. }
$$

If $\tilde{x}_{\theta}=a+x_{\theta}$, we have

$$
z \leq \tilde{x}_{\theta}<\tilde{x}_{\theta}+h_{0} \leq z+\ell_{0}
$$

Moreover,

$$
\left\{\begin{array}{l}
\left|f_{\theta}\left(\tilde{x}_{\theta}\right)-f_{\theta}\left(x_{\theta}\right)\right|=\left|\tau_{a} f_{\theta}\left(x_{\theta}\right)-f_{\theta}\left(x_{\theta}\right)\right| \leq \varepsilon \\
\left|f_{\theta}\left(\tilde{x}_{\theta}+h_{0}\right)-f_{\theta}\left(x_{\theta}+h_{0}\right)\right|=\left|\tau_{a} f_{\theta}\left(x_{\theta}+h_{0}\right)-f_{\theta}\left(x_{\theta}+h_{0}\right)\right| \leq \varepsilon,
\end{array}\right.
$$

and it follows that

$$
\frac{\left|f_{\theta}\left(\tilde{x}_{\theta}+h_{0}\right)-f_{\theta}\left(\tilde{x}_{\theta}\right)\right|}{\left|h_{0}\right|} \geq \frac{\left|f_{\theta}\left(x_{\theta}+h_{0}\right)-f_{\theta}\left(x_{\theta}\right)\right|-2 \varepsilon}{\left|h_{0}\right|} \geq \frac{(1+v)\left\|g^{\prime}\right\|_{\infty}}{b^{1-\alpha}-1} .
$$

We have just proved that $M_{h_{0}} \subset M_{h_{0}, \ell_{0}}$, and we can conclude that

$$
\mathbb{P}\left[M_{h_{0}, \ell_{0}}\right] \geq \mathbb{P}\left[M_{h_{0}}\right]>0 .
$$

We can now finish the proof of Theorem 3.1 Suppose that $g$ is not constant and let $v>0$. According to Lemma 4.2, choose $h_{0}>0$ and $\ell_{0} \geq 1$ such that

$$
\mathbb{P}\left[M_{h_{0}, \ell_{0}}\right]>0 .
$$

Using the ergodicity of the probability measure $\mathbb{P}$, we can claim that for almost every $\theta \in \Omega, T^{n}(\theta) \in M_{h_{0}, \ell_{0}}$ infinitely often (the set $\lim _{\sup _{n \rightarrow \infty}} T^{-n}\left(M_{h_{0}, \ell_{0}}\right)$ is $T$-invariant with positive probability).

Denote by $E$ this set of full measure. If $\theta \in E$, we can construct a sequence of integers $\left(n_{k}\right)_{k \geq 0}$ increasing to $+\infty$ such that

$$
T^{n_{k}}(\theta) \in M_{h_{0}, \ell_{0}} \quad \text { for all } k \geq 0 .
$$

Let $\delta_{k}=\ell_{0} b^{-n_{k}}$. If $I$ is an interval of length $|I|=\delta_{k}$ and if $\tilde{I}=b^{n_{k}} I$, we can find $x \in \tilde{I}$ such that $x+h_{0} \in \tilde{I}$ and

$$
\left|f_{T^{n_{k} \theta}}\left(x+h_{0}\right)-f_{T^{n_{k} \theta}}(x)\right| \geq \frac{(1+v)\left\|g^{\prime}\right\|_{\infty}}{b^{1-\alpha}-1}\left|h_{0}\right| .
$$


Iterating Lemma 4.1, we obtain

$$
\left|f_{\theta}\left(b^{-n_{k}}\left(x+h_{0}\right)\right)-f_{\theta}\left(b^{-n_{k}} x\right)\right| \geq \frac{\left(1+v b^{n_{k}(1-\alpha)}\right)\left\|g^{\prime}\right\|_{\infty}}{b^{1-\alpha}-1}\left|b^{-n_{k}} h_{0}\right| .
$$

Take

$$
C=\frac{v\left\|g^{\prime}\right\|_{\infty}\left|h_{0}\right|}{\ell_{0}^{\alpha}\left(b^{1-\alpha}-1\right)} .
$$

This constant does not depend on $\theta$ and $k$ and is such that

$\operatorname{osc}\left(f_{\theta}, I\right) \geq\left|f_{\theta}\left(b^{-n_{k}}\left(x+h_{0}\right)\right)-f_{\theta}\left(b^{-n_{k}} x\right)\right| \geq \frac{v b^{n_{k}(1-\alpha)}\left\|g^{\prime}\right\|_{\infty}}{b^{1-\alpha}-1}\left|b^{-n_{k}} h_{0}\right|=C|I|^{\alpha}$.

This completes the proof of Theorem 3.1

Let us now prove Theorem 3.2. As previously, we choose $v>0, h_{0}>0$ and $\ell_{0} \geq 1$ such that

$$
\mathbb{P}\left[M_{h_{0}, \ell_{0}}\right]>0 .
$$

We need more quantitative information about the sequence of iterates $T^{n_{k}}$ satisfying $T^{n_{k}}(\theta) \in M_{h_{0}, \ell_{0}}$. This will be given by the ergodic theorem, which gives us the asymptotic behavior of the frequency of the returns in $M_{h_{0}, \ell_{0}}$ (for a simple proof of the ergodic theorem, see for example [4], page 98). More precisely, this theorem states that almost surely,

$$
\frac{1}{n} \sum_{j=0}^{n-1} \mathbb{1}_{M_{h_{0}, \ell_{0}}} \circ T^{j} \underset{n \rightarrow \infty}{\longrightarrow} \mathbb{P}\left[M_{h_{0}, \ell_{0}}\right]
$$

In other words,

$$
\lim _{k \rightarrow \infty} \frac{n_{k}(\theta)}{k}=\frac{1}{\mathbb{P}\left[M_{h_{0}, \ell_{0}}\right]} \quad \text { almost surely, }
$$

where $n_{k}(\theta)$ is the $k$-th return in $M_{h_{0}, \ell_{0}}$.

Denote by $\tilde{E}$ the set of $\theta$ satisfying (4.2), and let $\varepsilon>0$. If $\theta \in \tilde{E}$, the sequence $n_{k}(\theta) / n_{k-1}(\theta)$ tends to 1 at infinity. So, we can find $k_{0} \geq 1$ such that

$$
\forall k \geq k_{0}, \quad \frac{n_{k}(\theta)}{n_{k-1}(\theta)} \leq \frac{\alpha+\varepsilon}{\alpha} .
$$

For simplicity, write $n_{k}$ instead of $n_{k}(\theta)$ and let $I$ be an interval of length $|I| \leq$ $\ell_{0} b^{-n_{k_{0}-1}}$. There exists $k \geq k_{0}$ such that

$$
\ell_{0} b^{-n_{k}} \leq|I| \leq \ell_{0} b^{-n_{k-1}} .
$$

We can then choose an interval $J \subset I$ with length $|J|=\ell_{0} b^{-n_{k}}$. Using Lemma 4.1 as in the proof of Theorem 3.1 we get

$$
\operatorname{osc}\left(f_{\theta}, I\right) \geq \operatorname{osc}\left(f_{\theta}, J\right) \geq \frac{\left(1+v b^{n_{k}(1-\alpha)}\right)\left\|g^{\prime}\right\|_{\infty}}{b^{1-\alpha}-1}\left|b^{-n_{k}} h_{0}\right| \geq \frac{v\left|h_{0}\right|\left\|g^{\prime}\right\|_{\infty}}{b^{1-\alpha}-1}\left|b^{-\alpha n_{k}}\right| .
$$

The choice of $k_{0}$ ensures that

$$
b^{-\alpha n_{k}} \geq \frac{1}{\ell_{0}^{\alpha+\varepsilon}}|I|^{\alpha+\varepsilon} .
$$

The conclusion follows for every interval $I$ of length $|I| \leq \ell_{0} b^{-n_{k_{0}-1}}$ if we put

$$
C=\frac{v\left|h_{0}\right|\left\|g^{\prime}\right\|_{\infty}}{\ell_{0}^{\alpha+\varepsilon}\left(b^{1-\alpha}-1\right)} .
$$

Finally, replacing $C$ by $\min \left(C, C\left(\ell_{0} b^{-n_{k_{0}-1}}\right)^{\alpha+\varepsilon}\right)$, we have the conclusion for any interval $I$ of length $|I| \leq 1$. 
Final remark on Theorem [3.2. With the method used in the proof of Theorem 3.2 we would have to know that $n_{k+1}(\theta)-n_{k}(\theta)$ is almost surely bounded in order to conclude that (3.3) is true when $\varepsilon=0$. Of course, this assertion is false: the number of iterates between two consecutive returns in $M_{h_{0}, \ell_{0}}$ cannot be uniformly bounded, and the ergodic theorem only ensures that $n_{k+1}(\theta) / n_{k}(\theta)$ tends to 1 with probability one.

\section{The CASE Where $b$ IS TRANSCEndental}

In the case where $b$ is a transcendental number, we can improve the conclusion of Theorems 3.1 and 3.2. This is the purpose of the following result.

Theorem 5.1. Let $b>1$ be a transcendental number, $0<\alpha<1$, and $g: \mathbb{R} \rightarrow \mathbb{R}$ a 1-periodic non-constant Lipschitz function. Define $f_{\theta}$ by formula (1.4). There exists a constant $C>0$ such that for every $\theta \in \Omega$ and for every interval $I$ of length $|I| \leq 1$,

$$
\operatorname{osc}\left(f_{\theta}, I\right) \geq C|I|^{\alpha} .
$$

Remark 5.2. In particular, the conclusion is true when $\theta=(0,0, \cdots)$. This seems to be in contradiction with the possible case (i) in Theorem [1.1. In fact, it means that if $f$ is almost periodic, Lipschitz and non-constant, and if $b$ is a transcendental number, then $g(x)=f(x)-b^{-\alpha} f(b x)$ cannot be 1-periodic. An elementary proof of this property can be obtained by Fourier analysis. For algebraic numbers, such a construction is possible. For example, if $b=\sqrt{2}$ and if $g(x)=\cos (2 \pi x)-$ $2^{-\alpha} \cos (4 \pi x)$, we obtain

$$
f(x)=\sum_{n=0}^{+\infty} b^{-n \alpha} g\left(b^{n} x\right)=\cos (2 \pi x)+2^{-\alpha / 2} \cos \left(2^{3 / 2} \pi x\right),
$$

which is a regular function.

Proof of Theorem [5.1. The proof of Theorem 5.1 is a consequence of the following lemma, which replaces Lemma 4.2

Lemma 5.3. In the notation of Lemma 4.2, suppose that $b$ is a transcendental number. Then, for all $v>0$, there exist $h_{0}>0$ and $\ell_{0}>0$ such that

$$
M_{h_{0}, \ell_{0}}=\Omega \text {. }
$$

Proof. Remember the notation in the proof of Lemma 4.2 and let $h_{0}$ be a positive number such that $\mathbb{P}\left[M_{h_{0}}\right]>0$. Let $t \neq 0$ and define the translation $S: \Omega \rightarrow \Omega$ by

$$
(S \theta)_{n}=\theta_{n}+t b^{n}(\bmod 1) .
$$

We know that in the compact group $(\mathbb{R} / \mathbb{Z})^{\mathbb{N}}$ (equipped with the Haar measure), the translation by an element $\lambda=\left(\lambda_{n}\right)_{n \geq 0}$ is ergodic if and only if the real numbers $1, \lambda_{0}, \cdots, \lambda_{n}, \cdots$ are $\mathbb{Q}$-independent (it suffices to note that invariant $L^{2}$ functions $f$ are exactly those whose Fourier coefficients satisfy $\hat{f}\left(n_{0}, \cdots, n_{p}\right)=$ $e^{2 i \pi\left(n_{0} \lambda_{0}+\cdots+n_{p} \lambda_{p}\right)} \hat{f}\left(n_{0}, \cdots, n_{p}\right)$ for every $\left.p \geq 0\right)$. When $b$ is a transcendental number, we can then conclude that $S$ is $\mathbb{P}$-invariant and ergodic. On the other hand, as an easy consequence of the relation $f_{S \theta}(x-t)=f_{\theta}(x)$, we note that $M_{h_{0}}$ is invariant under the translation $S$. It follows that $\mathbb{P}\left[M_{h_{0}}\right]=1$. If $\ell_{0}$ is constructed as in Lemma 4.2, we get $\mathbb{P}\left[M_{h_{0}, \ell_{0}}\right]=1$. In particular, $M_{h_{0}, \ell_{0}}$ is dense in $\Omega$. Moreover, if a sequence $\left(\theta^{n}\right)_{n \geq 0}$ of points of $M_{h_{0}, \ell_{0}}$ converges to $\theta \in \Omega$, we know that 
the sequence $f_{\theta^{n}}$ converges uniformly to $f_{\theta}$. Using the compactness of the intervals $I=\left[z, z+\ell_{0}\right]$ of length $\ell_{0}$, it is then easy to conclude that $\theta \in M_{h_{0}, \ell_{0}}$. Finally, $M_{h_{0}, \ell_{0}}$ is dense and closed in $\Omega$. This means that $M_{h_{0}, \ell_{0}}=\Omega$.

Remark 5.4. Let $B=\left(b^{n}\right)_{n \geq 0}$ with $b$ a transcendental number. For every $\theta \in \Omega$, the projection of the curve $(\theta+t B)_{t \in \mathbb{R}}$ in the infinite-dimensional torus $(\mathbb{R} / \mathbb{Z})^{\mathbb{N}}$ is dense in $(\mathbb{R} / \mathbb{Z})^{\mathbb{N}}$. If we can prove that $M_{h_{0}} \neq \emptyset$ for some $h_{0}>0$, we can conclude that $M_{h_{0}}$ is dense in $\Omega$ and then obtain another proof of Lemma 5.3. In fact, it is easy to construct $\theta \in \Omega$ and $h_{0}>0$ such that $\theta \in M_{h_{0}}$. Let $x_{0} \in[0,1]$ be a point where $g$ is minimal, and let $y_{0}>0$ be such that $g\left(y_{0}+x_{0}\right)>g\left(x_{0}\right)$. Let $\theta=\left(x_{0}, x_{0}\right.$, $\left.\cdots, x_{0}, \cdots\right)$. We have

$$
\begin{aligned}
f_{\theta}\left(b^{-p} y_{0}\right)-f_{\theta}(0) & =\sum_{n=0}^{+\infty} b^{-n \alpha}\left[g\left(b^{n-p} y_{0}+x_{0}\right)-g\left(x_{0}\right)\right] \\
& \geq b^{-p \alpha}\left[g\left(y_{0}+x_{0}\right)-g\left(x_{0}\right)\right] \\
& \geq \frac{(1+3 v)\left\|g^{\prime}\right\|_{\infty}}{b^{1-\alpha}-1}\left|b^{-p} y_{0}\right| \text { if } p \text { is sufficiently large, }
\end{aligned}
$$

and we can conclude that $\theta \in M_{b^{-p} y_{0}}$.

Let us now sketch the end of the proof of Theorem 5.1. The main argument is the one used for the previous proofs. Let $\theta \in \Omega$, and let $I$ be an interval such that $\ell_{0} b^{-k} \leq|I| \leq \ell_{0} b^{-k+1}$. An iteration of Lemma 5.3 gives

$$
\operatorname{osc}\left(f_{\theta}, I\right) \geq \operatorname{osc}\left(f_{\theta}, J\right) \geq \frac{\left(1+v b^{k(1-\alpha)}\right)\left\|g^{\prime}\right\|_{\infty}}{b^{1-\alpha}-1}\left|b^{-k} h_{0}\right| \geq \frac{v\left|h_{0}\right|\left\|g^{\prime}\right\|_{\infty}}{b^{1-\alpha}-1}\left[\frac{|I|}{\ell_{0} b}\right]^{\alpha},
$$

where $J \subset I$ has length $\ell_{0} b^{-k}$. We can choose $C=\frac{v\left|h_{0}\right|\left\|g^{\prime}\right\|_{\infty}}{\left(b^{1-\alpha}-1\right)\left(\ell_{0} b\right)^{\alpha}}$.

\section{ON FRACTAL DIMENSION OF THE GRAPH OF $f_{\theta}$}

Theorems 3.2 and 5.1 allow us to estimate the box-counting dimension and the packing dimension of the graph of $f_{\theta}$.

Theorem 6.1. Let $g: \mathbb{R} \rightarrow \mathbb{R}$ be a 1-periodic non-constant Lipschitz function, and let $1<b<+\infty$ and $0<\alpha<1$. Define $f_{\theta}$ by formula (1.4) and $\Gamma_{\theta}(A)$ by

$$
\Gamma_{\theta}(A)=\left\{\left(x, f_{\theta}(x)\right), x \in A\right\} .
$$

We have

(i) for every non-trivial compact interval $I, \Delta\left(\Gamma_{\theta}(I)\right)=2-\alpha$ almost surely and

(ii) for every non-trivial interval I, Dim $\left(\Gamma_{\theta}((I))=2-\alpha\right.$ almost surely, where $\Delta(E)$ and $\operatorname{Dim}(E)$ are respectively the box-counting dimension and the packing dimension of a set $E$.

Moreover, when $b$ is a transcendental number, conclusions (i) and (ii) are valid for every $\theta \in \Omega$.

Statement (i) is well known if we have a minoration of the oscillations (see, for example, [3]). The minoration of the packing dimension is probably less known (for general information about the packing dimension, see [18] or [17]). We can state the following elementary proposition. 
Proposition 6.2. Let $0<\gamma<1$, and let $h: \mathbb{R} \rightarrow \mathbb{R}$ be a function such that

$$
\operatorname{osc}(h, I) \geq C|I|^{\gamma} \quad \text { for all intervals } I \text { of length }|I| \leq 1 .
$$

Then, for every interval I with nonempty interior,

$$
\operatorname{Dim}(\Gamma(I)) \geq 2-\gamma
$$

where $\Gamma(A)=\{(x, h(x)), x \in A\}$.

Proof. We can suppose that $I=[a, b]$ is a compact interval with $a \neq b$ (the quantity Dim is $\sigma$-stable). Remember that if $E \subset \mathbb{R}^{2}$, then

$$
\operatorname{Dim}(E)=\inf \left\{\sup \Delta\left(E_{i}\right) ; E \subset \bigcup_{i=1}^{\infty} E_{i} \text { where the } E_{i} \text { are closed sets }\right\} .
$$

Suppose that $\Gamma([a, b]) \subset \bigcup_{i=1}^{\infty} E_{i}$. We can then find a sequence $\left(F_{i}\right)$ of closed subsets of $[a, b]$ with

$$
[a, b]=\bigcup_{i=1}^{\infty} F_{i} \quad \text { and } \quad \forall i \geq 1, \Gamma\left(F_{i}\right) \subset E_{i} .
$$

Baire's property ensures that we can find $i_{0}$ such that the interior of $F_{i_{0}}$ is not empty. It follows that $\Delta\left(E_{i_{0}}\right) \geq \Delta\left(\Gamma\left(F_{i_{0}}\right)\right) \geq 2-\gamma$, and we can conclude that $\operatorname{Dim}(\Gamma([a, b])) \geq 2-\gamma$.

\section{ACKNOWLEDGMENTS}

The author wants to thank Claude Tricot for helpful discussions about Weierstrass functions with phases. Thanks also to Guillaume Havard for his interest in this work and for his careful reading of the first drafts.

\section{REFERENCES}

1. T. Bousch and Y. Heurteaux, On oscillations of Weierstrass-type functions, manuscript, 1999.

2. Caloric measure on domains bounded by Weierstrass-type graphs, Ann. Acad. Sci. Fenn. 25 (2000), 501-522. MR 2001h:31004

3. K. Falconer, Fractal Geometry : Mathematical Foundations and Applications, John Wiley \& Sons, New York, 1990. MR 92j:28008

4. _ Techniques in fractal geometry, John Wiley \& Sons, New York, 1997. MR 99f:28013

5. G. H. Hardy, Weierstrass's non-differentiable function, Trans. Amer. Math. Soc. 17 (1916), 301-325.

6. T.-Y. Hu and K.-S. Lau, The sum of Rademacher functions and Hausdorff dimension, Math. Proc. Cambridge Philos. Soc. 108 (1990), 97-103. MR 91d:28020

7. , Fractal dimensions and singularities of the Weierstrass type functions, Trans. Amer. Math. Soc. 335 (1993), 649-665. MR 93d:28011

8. B. R. Hunt, The Hausdorff dimension of graphs of Weierstrass functions, Proc. Amer. Math. Soc. 126 (1998), 791-800. MR 98i:28009

9. J. L. Kaplan, J. Mallet-Paret, and J. A. Yorke, The Lyapunov dimension of a nowhere differentiable attracting torus, Ergodic Theory \& Dynamical Systems 4 (1984), 261-281. MR 86h:58091

10. F. Ledrappier, On the dimension of some graphs, Contemp. Math. 135 (1992), 285-293. MR 94d:28007

11. R. D. Mauldin and S. C. Williams, On the Hausdorff dimension of some graphs, Trans. Amer. Math. Soc. 298 (1986), 793-804. MR 88c:28006

12. C. McMullen, The Hausdorff dimension of general Sierpinski carpets, Nagoya Math. J. 96 (1984), 1-9. MR 86h:11061

13. K. Petersen, Ergodic theory, Cambridge University Press, Cambridge, 1983. MR 87i:28002

14. F. Przytycki and M. Urbanski, On the Hausdorff dimension of some fractal sets, Studia Math. 93 (1989), 155-186. MR 90f:28006 
15. Y. Shiota and T. Sekiguchi, Hausdorff dimension of graphs of some Rademacher series, Japan J. Appl. Math. 7 (1990), 121-129. MR 91e:28009

16. J. Szulga, Hausdorff dimension of Weierstrass-Mandelbrot process, Statist. Probab. Lett. 56 (2002), 301-307. MR 2002m:60069

17. C. Tricot, Sur la classification des ensembles boréliens de mesure de Lebesgue nulle, Ph.D. thesis, Faculté des Sciences de l'Université de Genève, 1980.

18. (1982), 57-74. MR 84d:28013

19. P. Walters, An introduction to ergodic theory, Springer-Verlag, New York, 1982. MR 84e:28017

Laboratoire de Mathématiques pures, Université Blaise Pascal, F-63177 Aubière Cedex, France

E-mail address: Yanick.Heurteaux@math.univ-bpclermont.fr 